\title{
Interactions and two-phase coexistence in nonionic micellar solutions as determined by static light scattering
}

\author{
Dzina Kleshchanok, ${ }^{a}$ Hartwig Strunk ${ }^{b}$ Remco Tuinier $^{a}$ and Peter R. Lang ${ }^{* a}$ \\ Received 16th September 2005, Accepted 22nd November 2005 \\ First published as an Advance Article on the web 9th December 2005 \\ DOI: $10.1039 / \mathbf{b 5 1 3 2 2 5 h}$
}

\begin{abstract}
We suggest semi-phenomenological approaches for the pair interaction potential in aqueous $\mathrm{C}_{m} \mathrm{E}_{n}$ solutions. These expressions are non-linear least squares fitted to static light scattering data from solutions of $\mathrm{C}_{m} \mathrm{E}_{4}$ and $\mathrm{C}_{m} \mathrm{E}_{8}$ surfactants in the long wavelength limit. From the resulting interaction parameters we calculate the location of the liquid/liquid two phase coexistence curves, which are in very good agreement with experimental data reported by others.
\end{abstract}

\section{Introduction}

Aqueous solutions containing nonionic surfactants of the oligo(ethyleneoxide)- $n$-alkylether family $\left(\mathrm{C}_{m} \mathrm{E}_{n}\right)$ have been studied thoroughly for almost half a century. ${ }^{1,2} \mathrm{~A}$ great effort has been dedicated to the accurate determination of the mixtures' phase diagrams in the temperature vs. composition plane. ${ }^{3-5}$ As a common feature all aqueous $\mathrm{C}_{m} \mathrm{E}_{n}$-surfactant solutions show an upper miscibility gap at elevated temperatures where two liquid phases coexist. The investigation of the micellar shape in isotropic solutions by scattering experiments was also a subject of continuous scientific interest throughout the last few decades. ${ }^{6-17}$ In all studies it was observed that the intensity scattered from a surfactant solution at constant composition increases drastically with increasing temperature, i.e. on approaching the coexistence curve. Further, a complex concentration dependence of the long wave length limit of the scattered intensity $I(Q \rightarrow 0)$ was observed, which is proportional to the concentration-dependent structure factor at zero scattering vector, $S(Q=0)$. For most of the systems investigated $I(Q \rightarrow 0)$ goes through a distinct maximum as a function of the micellar volume fraction. There are however systems where a monotonous decrease of $S(Q=0)$ is observed with increasing surfactant content. It has been a matter of a longstanding discussion whether these effects are due to micellar growth, due to increasing attractive inter-micellar interaction, or solely due to critical opalescence. In the earlier days most authors were taking up rather puristic positions. Several groups claimed that critical fluctuations are dominating the scattering behavior and that structural properties of the micelles are negligible. ${ }^{18-21}$ On the other hand, Hayter et al. ${ }^{6}$ and Zulauf et al. ${ }^{22}$ interpreted small-angle neutron and light scattering experiments on aqueous $\mathrm{C}_{8} \mathrm{E}_{4}$ and $\mathrm{C}_{8} \mathrm{E}_{5}$ solutions using a model of spherical micelles with increasing attractive interaction, where micellar growth is explicitly excluded. Lindman and colleagues meanwhile insisted on the opinion that nonionic micelles grow with temperature. ${ }^{23}$ However, NMR

\footnotetext{
${ }^{a}$ Forschungszentrum Jülich, Institut für Festkörperforschung, Weiche Materie, 52425 Jülich, Germany.E-mail: p.lang@fz-juelich.de

${ }^{b} 64297$, Darmstadt
}

studies by the same group demonstrated there is no indication for micellar growth in the $\mathrm{C}_{8} \mathrm{E}_{4}$ /water system. ${ }^{24}$

In the 1980s there was already experimental evidence that different temperature-dependent phenomena can be observed in the same system. Cebula et al. interpreted small-angle neutron scattering data from $\mathrm{C}_{12} \mathrm{E}_{6}$ solution with a model of cylindrical micelles, growing in length with temperature, ${ }^{25}$ while Strey et al. found for the same system that globular micelles exist at low temperatures, which transform into large aggregates at elevated temperatures, and finally at even higher temperatures critical fluctuations become dominant. ${ }^{26}$

Due to improvements both in experimental instrumentation and interpretation ${ }^{14}$ of small-angle neutron scattering, Glatter et al. showed conclusively that micellar growth and increasing attractive interaction have to be considered to describe the observed scattering data. ${ }^{16}$ In that contribution it is pointed out that the importance of micellar growth varies with the relative length of the hydrophilic and the hydrophobic moieties. There are systems such as $\mathrm{C}_{12} \mathrm{E}_{5}$ which form cylindrical micelles throughout the entire temperature range, as well as systems which form spherical micelles at low temperatures that transform into cylindrical micelles on increasing temperature. The tendency to form cylindrical micelles decreases with increasing length of the hydrophilic chains at constant length of the hydrophobic part (Israelachvili's rule of thumb ${ }^{27}$ ). Accordingly, $\mathrm{C}_{12} \mathrm{E}_{6}$ forms spherical micelles below $10{ }^{\circ} \mathrm{C}$ and cylindrical aggregates only at elevated temperatures. In those contributions, it is shown that micellar interaction leads to a solution structure factor, $S(Q)$, which has to be considered in the data evaluation procedure. However, the authors make no attempt to further quantify the nature of the interaction.

To this end there are only few attempts in literature towards a thourough description of the particle interaction. Schurtenberger and Cavaco used the expression from renormalization group theory for $S(Q=0)$ of polymers with excluded volume $\mathrm{e}^{29}$ to describe the light scattering data from cylindrical micelles quantitatively. ${ }^{28}$ This model holds for $\mathrm{C}_{m} \mathrm{E}_{n}$ surfactants which form very long cylindrical micelles like $\mathrm{C}_{16} \mathrm{E}_{6}{ }^{13}$ or $\mathrm{C}_{12} \mathrm{E}_{5} \cdot{ }^{15}$ Hayter et al. ${ }^{6}$ proposed the combination of a short ranged attraction and a hard core repulsion potential. They chose a Yukawa-type attraction for mathematical convenience. This model has been of limited use for predicting the 
temperature dependence of the scattering intensity. However, it fails to correctly reproduce the measured concentration dependence of $S(Q=0)$. Another model which combines the repulsion of a hard core volume with a short ranged attractive potential, is the Baxter sticky sphere model. ${ }^{30}$ Although this model was applied with great success to oil swollen micellar systems ${ }^{31,32}$ it was, to our knowledge, not used for the description of binary $\mathrm{C}_{m} \mathrm{E}_{n}$ /water solutions.

From thermodynamics it follows that $S(Q=0)$ is related to the osmotic compressibility and hence represents the collective long wavelength limit behavior of the system. Changes in $S(Q=0)$ reflect the many-body interactions between the micelles. Here we show that a proper equation of state, based on the pair interaction potential between the micelles allows to describe the experimental $S(Q=0)$ data accurately and enables to predict the location of the liquid/liquid phase coexistence curve. We report on static light scattering data from five $\mathrm{C}_{m} \mathrm{E}_{n} /$ water systems, two of which $\left(\mathrm{C}_{6} \mathrm{E}_{4}\right.$ and $\left.\mathrm{C}_{8} \mathrm{E}_{4}\right)^{24,33}$ are likely to form spherical micelles or anisometric micelles with low aspect ratio. Two other systems where chosen by doubling $m$ and $n\left(\mathrm{C}_{12} \mathrm{E}_{8}\right.$ and $\left.\mathrm{C}_{16} \mathrm{E}_{8}\right)$ and finally we chose a fifth in-between these two sets, namely $\mathrm{C}_{10} \mathrm{E}_{8}$. The surfactants can be divided into two groups according to their scattering behavior. The $S(Q=0) v s$. volume fraction curves of the $\mathrm{C}_{m} \mathrm{E}_{4}$-group run through a pronounced maximum, the height of which increases with temperature, while for the $\mathrm{C}_{m} \mathrm{E}_{8}$ group $S(Q=0)$ decreases monotonously with increasing volume fraction of micelles. From this fact it is immediately obvious that the two groups require different approaches for the micellar interaction to describe $S(Q=0)$ correctly.

For the first $\left(\mathrm{C}_{6} \mathrm{E}_{4}\right.$ and $\left.\mathrm{C}_{8} \mathrm{E}_{4}\right)$ set we designed a semiphenomenological approach for the pair interaction potential which, like former approaches, contains the superposition of a repulsive and an attractive part. The repulsion consists of a hard core excluded volume term with an additional steric contribution and the attraction is described as a Van der Waals term. Since it is rather involving to formulate interaction potentials for anisometric particles we chose to account for possible micellar growth by rescaling the volume fraction with an effective micellar size and an effective aggregation number, while still assuming spherical symmetry of the interaction potential. For the second group $\left(\mathrm{C}_{10} \mathrm{E}_{8}, \mathrm{C}_{12} \mathrm{E}_{8}\right.$ and $\mathrm{C}_{12} \mathrm{E}_{8}$ ) we modified a Flory-Huggins type description for the chemical potential and the osmotic pressure of cylindrical micelles such that we could calculate the osmotic compressibility, which is related to $S(Q=0)$. In both cases non-linear least squares fitting of the model expressions to the data allowed for the determination of interaction parameters, which in turn were used to calculate the two-phase coexistence curves of the phase diagrams. In both cases the experimental and the calculated coexistence curves agree well.

\section{Theoretical models}

\subsection{Interaction and equation of state of spherical micelles}

The micelles are modeled as consisting of spherical hydrophobic cores with volume $V_{\mathrm{s}}$, which interact via an attractive Van der Waals potential $W_{\mathrm{vdW}}$, and a hydrophilic corona, which acts as a polymer brush of height $H_{\mathrm{b}}$ that stabilizes the micelles against aggregation via steric brush repulsion $W_{\mathrm{b}}$. Apart from the excluded volume interaction we assume these two contributions to dominate the system. Jansen et al. ${ }^{34}$ considered these interactions to interpret the phase behavior of sticky sterically stabilized silica spheres, where they applied the Percus-Yevick expression $^{35}$ for the excluded volume contribution. As the Carnahan-Starling ${ }^{36}$ approach yields a more accurate results for hard spheres, ${ }^{37}$ we followed the procedure by Vrij et al. ${ }^{38}$ who used the Carnahan-Starling-Van der Waals equation of state for the description of spherical microemulsions

$$
\frac{\Pi V_{\mathrm{s}}}{k_{\mathrm{B}} T}=\phi \frac{1+\phi+\phi^{2}-\phi^{3}}{(1-\phi)^{3}}-\gamma \phi^{2},
$$

where $\phi$ is the micellar volume fraction. On the right-hand side, the first term represents the contribution of the hard sphere excluded volume according to Carnahan-Starling, and the second term incorporates all non-hard sphere interactions. Comparison of the volume fraction expansion of eqn (1.1) up to second order

$$
\frac{\Pi V_{\mathrm{s}}}{k_{\mathrm{B}} T}=\phi(1+(4-\gamma) \phi+\ldots)
$$

with a simple general virial expansion

$$
\frac{\Pi V_{\mathrm{s}}}{k_{\mathrm{B}} T}=\phi\left(1+B_{2} \phi \ldots\right)
$$

shows that the Van der Waals parameter $\gamma$ can be identified as $4-B_{2}$. Here $B_{2}$ is the normalized second osmotic virial coefficient, which follows from statistical mechanics as

$$
B_{2}=\frac{2 \pi}{V_{\mathrm{s}}} \int_{0}^{\infty} r^{2}\left(1-\exp \left\{-\frac{W_{\text {tot }}(r)}{k_{\mathrm{B}} T}\right\}\right) \mathrm{d} r,
$$

where $r$ is the center-to-center distance between two particles. The corresponding expression for $\gamma$ is

$$
\gamma=\frac{2 \pi}{V_{\mathrm{s}}} \int_{2 a}^{\infty} r^{2}\left(\exp \left\{-\frac{W_{\mathrm{tot}}(r)}{k_{\mathrm{B}} T}\right\}-1\right) \mathrm{d} r,
$$

where $a$ is the particle radius and the interaction potential is infinitely repulsive for $0 \leq r \leq 2 a$. In the superposition approximation we write $W_{\text {tot }}(r)=W_{\mathrm{vdw}}(r)+W_{\mathrm{b}}(r)$ with

$$
\frac{W_{\mathrm{vdW}}(r)}{k_{\mathrm{B}} T}=-\frac{A_{\mathrm{H}}}{6}\left[\frac{2 a^{2}}{r^{2}-4 a^{2}}+\frac{2 a^{2}}{r^{2}}+\ln \left(\frac{r^{2}-4 a^{2}}{r^{2}}\right)\right]
$$

where $A_{\mathrm{H}}$ is the Hamaker constant. ${ }^{39}$ As the Van der Waals attraction diverges at contact, we introduced a cut-off at $r=2 a$ $+a \times 10^{-2}$. According to the Alexander-DeGennes theory, ${ }^{40,41}$ the brush repulsion in the Derjaguin approximation is ${ }^{42}$

$$
\begin{aligned}
\frac{W_{\mathrm{b}}(r)}{k_{\mathrm{B}} T} & =\frac{16 \pi a H_{\mathrm{b}}^{2} \sigma^{\frac{3}{2}}}{35}\left[28\left(\left(\frac{2 H_{\mathrm{b}}}{r-2 a}\right)^{\frac{1}{4}}-1\right)\right. \\
& \left.+\frac{20}{11}\left(1-\left(\frac{r-2 a}{2 H_{\mathrm{b}}}\right)^{\frac{11}{4}}\right)+12\left(\frac{r-2 a}{2 H_{\mathrm{b}}}-1\right)\right]
\end{aligned}
$$

in the range $2 a<r \leq 2\left(a+H_{\mathrm{b}}\right)$. For $r \leq 2 a$ the interaction is infinitely repulsive and for $r>2\left(a+H_{\mathrm{b}}\right)$ the brush repulsion vanishes. The brush height is related to the contour length, $L_{\mathrm{c}}$, 
of the corona chains, the surface area fraction they cover, $\theta$, and their segments' normalized excluded volume, $v$, by

$$
H_{\mathrm{b}}=L_{\mathrm{c}}\left(\frac{8 v \theta}{\pi^{2}}\right)^{\frac{1}{3}}
$$

Here the covered area fraction $\theta$ is dimensionless. It is given by $\theta=\sigma \pi\left(d_{\mathrm{c}} / 2\right)^{2}$ where $\pi\left(d_{\mathrm{c}} / 2\right)^{2}$ is the surface area covered by a single segment and $\sigma$ is the grafting density expressed as number of brush chains per area.

The brush repulsion is a temperature-dependent interaction potential, since the normalized excluded volume per segment is related to the Flory-Huggins parameter as $v=1-2 \chi$, which for PEO chain depends on temperature as $\chi(\vartheta)=6.2528 \times$ $10^{-3} \vartheta-0.085485 .^{43}$ where $v$ is the temperature in ${ }^{\circ} \mathrm{C}$.

The long wavelength limit of the solution structure factor $S(Q=0)$ is related to the osmotic compressibillity, which can be easily calculated as the first derivative of eqn (1.1)

$$
\begin{aligned}
S(Q=0) & =k_{\mathrm{B}} T \frac{\partial \phi}{\partial \Pi V} \\
& =\left(\frac{(1+2 \phi)^{2}+\phi^{3}(\phi-4)}{(1-\phi)^{4}}-2 \gamma \phi\right)^{-1} .
\end{aligned}
$$

Using the Van der Waals/Carnahan-Starling equation of state (eqn (1.1)), expressions can be derived for the chemical potential, $\mu$, and the Helmholtz free energy $f=\phi \mu / k_{\mathrm{B}} T+$ $V_{\mathrm{s}} \Pi / k_{\mathrm{B}} T$ where $f \equiv F V_{\mathrm{s}} / k_{\mathrm{B}} T V$ is the Helmholtz free energy, $F$, normalized with $k_{\mathrm{B}} T$, the volume of a single sphere, $V_{\mathrm{s}}$, and the total volume of the system, $V$. From the equation of state follows the normalized Helmholtz energy

$$
f=\phi \ln \phi-\phi+\frac{4 \phi^{3}-3 \phi^{2}}{(1-\phi)^{3}}-\gamma \phi^{2} .
$$

If $\gamma$ is known, eqn (1.10) can be used to numerically calculate the location of the two-phase coexistence curve from the conditions $\mu_{1}=\mu_{2}$ and $\Pi_{1}=\Pi_{2}{ }^{44}$ Here $\mu_{j}$ and $\Pi_{j}$ represent the chemical potential and the osmotic pressure of the spheres in phase $j$.

\subsection{Interaction and thermodynamic properties of cylindrical micelles}

Rupert ${ }^{45}$ proposed that micellar $\mathrm{C}_{m} \mathrm{E}_{n}$ systems could be described using a modified Flory-Huggins model. He suggested an analogy between a cylindrical micelle and a polymer chain. Each surfactant molecule in a micelle represents a segment in a polymer chain and the number of segments per chain, $N$, then automatically represents the micellar aggregation number. The chemical potential of the water molecules $\mu_{\mathrm{w}}$ then reads:

$$
\mu_{\mathrm{w}}=\mu_{\mathrm{w}}^{0}+\frac{R T}{\beta}\left[\ln (1-\phi)+\left(1-\frac{1}{N}\right) \phi+\chi_{\mathrm{wm}} \phi^{2}\right],
$$

where $\mu_{\mathrm{w}}^{0}$ is the chemical potential of water in pure water, $\beta$ is the ratio of the volume of a surfactant over the volume of a solvent molecule (water), and $\chi_{w m}$ is the Flory-Huggins interaction parameter between micellar surfactant molecules $\mathrm{m}$ and water molecules w, consisting of enthalpic, $H_{\mathrm{wm}}$, and entropic
$S_{\mathrm{wm}}$ components:

$$
\chi_{\mathrm{wm}}=\frac{S_{\mathrm{wm}}}{R}-\frac{H_{\mathrm{wm}}}{R T} .
$$

The osmotic pressure of the micelles in a Flory-Huggins approach follows from:

$$
\Pi v_{\mathrm{w}}=\mu_{\mathrm{w}}^{0}-\mu_{\mathrm{w}}
$$

so,

$$
\Pi v_{w}=-\frac{R T}{\beta}\left[\ln (1-\phi)+\left(1-\frac{1}{N}\right) \phi+\chi_{\mathrm{wm}} \phi^{2}\right] .
$$

The first derivative of the osmotic pressure $\partial \Pi / \partial \phi$ follows as:

$$
\frac{\partial \Pi v_{\mathrm{w}}}{\partial \phi}=\frac{R T}{\beta}\left[\frac{1}{1-\phi}+\frac{1}{N}-1-2 \chi_{\mathrm{wm}} \phi\right] .
$$

As in Section 1.1.1 we calculate $S(Q=0)$, from the osmotic compressibility which for our case yields

$$
S(Q=0)=\beta\left[\frac{1}{1-\phi}+\frac{1}{N}-1-2 \chi_{\mathrm{wm}} \phi\right]^{-1} .
$$

Note that for $\phi \rightarrow 0, S(Q=0)$ approaches $\beta N$ and can thus be used to determine the molar mass in the limit of infinite dilution. In case of stronger attractions, which means higher temperatures for $\mathrm{C}_{m} \mathrm{E}_{n}$ systems, $\chi_{\mathrm{wm}}$ increases, so the structure factor is expected to increase in such a case. Above about $\chi_{\mathrm{wm}}=0.5$ one expects demixing in two phases. The coexistence curve can be calculated if $\chi_{\mathrm{wm}}$ is known, since the chemical potential of the micellar particles follows from Flory-Huggins theory as:

$\mu_{\mathrm{m}}=\mu_{\mathrm{m}}^{0}+R T\left[\ln (\phi)+(1-N)(1-\phi)+N \chi_{\mathrm{wm}}(1-\phi)^{2}\right]$

and the location of the coexistence curve is determined by the requirement that the chemical potential of each component is equal in both phases, i.e. $\mu_{\mathrm{m}, 1}=\mu_{\mathrm{m}, 2}$ and $\mu_{\mathrm{w}, 1}=\mu_{\mathrm{w}, 2}$.

\section{Experimental}

\subsection{Samples and preparation}

The surfactants were obtained from Sigma-Aldrich Co., Germany with a quoted purity of $98 \%$ and used without further purification. As a solvent we used ultrapure Milli-Q water (resistivity better than $18.2 \mathrm{M} \Omega \mathrm{cm}^{-1}$; Millipore $\mathrm{GmbH}$, Germany) for all experiments and cleaning steps. Aqueous solutions of the surfactants were prepared by weight and filtered three times (Rotilabo filters, Nylon, $0.45 \mu \mathrm{m}$; Carl Roth $\mathrm{GmbH}+\mathrm{Co}$., Germany). The concentrations used for data evaluation were corrected with critical micellar concentrations (cmc) using the literature values. ${ }^{46}$ All surfactant concentrations used in the measurements were much higher than $\mathrm{cmc}$ and belong to the isotropic region.

\subsection{Light scattering}

Measurements were performed with two different set-ups. First, a commercial ALV instrument (ALV-Laservertiebsgesellschaft, Langen, Germany) for simultaneous static and 
dynamic light scattering, which has been described in detail ${ }^{15}$ and second, a home-built scattering set-up which was optimized for static scattering experiments. The latter applying the same optical path as the classical Fica instruments. ${ }^{47}$ As light sources we used an Ar-ion laser at $\lambda_{0}=488 \mathrm{~nm}$, type Innova 70-2 from Coherent with the ALV instrument, and a mercury lamp at $\lambda_{0}=546 \mathrm{~nm}$ (Oriel) with the home-built set-up.

Averaged scattered intensities were recorded in a range of scattering angles $30 \leq \theta \leq 150^{\circ}$ which corresponds to a range of scattering vectors $8 \times 10^{4} \mathrm{~cm}^{-1} \lesssim Q=4 \pi n / \sin (\theta / 2) / \lambda_{0} \lesssim$ $3 \times 10^{5} \mathrm{~cm}^{-1}$ varying with the wavelength and the index of refraction, $n$. The raw data were brought to absolute scale in terms of the so-called Raleigh ratio $R(Q)$ applying standard procedures $^{48}$ and using p.a. grade toluene as a reference scatterer. The latter was also used to check the alignment of the instruments, and it was always found that the scattered intensity from pure toluene was constant within $\pm 3 \%$ over the whole angular range when normalized to the scattering volume. Since the intensity scattered from the solvent was very small in comparison with the intensities scattered from solutions it could be neglected for calculation of the Rayleigh ratio. The scattering contrast $K=4 \pi^{2}(\partial n / \partial c)^{2} n_{\text {solv }}{ }^{2} /\left(N_{\mathrm{A}} \lambda_{0}{ }^{4}\right)$ was calculated from the refractive index increments, $(\partial n / \partial c)$, published by Matsumoto ${ }^{49}$ at 298 K. For other temperatures $(\partial n / \partial c)$ values were interpolated from the data given by Balmbra. ${ }^{2}$ The temperature dependence of the refractive index of the solvent has been accounted for using an appropriate expression from literature. ${ }^{48}$

The Rayleigh ratios were detected for each solution and converted into values of $K c / R(Q, c)$ at fixed concentration $c$. These were plotted vs. $Q^{2}$ and approximated with a linear least squares fit. The linear extrapolation yields as the intercept a quantity which is usually referred to as the inverse apparent molar mass $\lim _{Q \rightarrow 0} K c / R(Q, c)=1 / M_{\text {app}}$, with $M_{\text {app }}=$ $M_{\mathrm{w}} S(Q=0)$ where $M_{\mathrm{w}}$ is the mass-averaged molar mass of the solute particles. The concentration dependence of $M_{\text {app }}$ at fixed temperature was fitted with the theoretical expression derived in sections 2.1 and 2.2, with $M_{\mathrm{w}}=M_{\text {mono }} N$ as a floating parameter, where $M_{\text {mono }}$ is the molar mass of the monomeric surfactant.

\section{Results and discussion}

\subsection{Experimental findings}

The concentration dependence of the scattered intensity from solutions of $\mathrm{C}_{6} \mathrm{E}_{4}$ recorded at $283 \mathrm{~K}$ follows perfectly the Carnahan-Starling prediction for hard spheres, ${ }^{36}$ i.e. eqn (1.9) with $\gamma=0$ as is shown in the inset of Fig. 1, where we have plotted $M_{\text {app }}$ vs. c. However, at higher temperatures these curves show a pronounced maximum at $c \gtrsim 0.1 \mathrm{~g} \mathrm{~mL}^{-1}$, the height of which increases with increasing temperature. This behavior can be phenomenologically interpreted as the balance between an attractive interaction which causes the scattering intensity to increase with increasing concentration and the repulsive hard core interaction potential which damps density fluctuations with increasing concentration. At high concentrations the repulsive part dominates the system and the scattered intensity decreases with increasing concentration. In

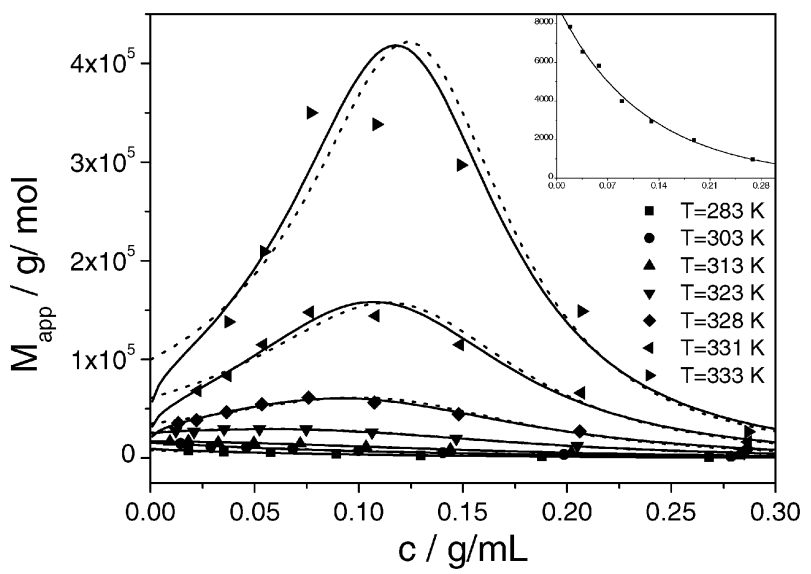

Fig. 1 Static light scattering data from solutions of $\mathrm{C}_{6} \mathrm{E}_{4}$ in water as $M_{\text {app }}$ vs. $c$ (symbols) recorded at temperatures as indicated in the legend. The dotted lines are linear least squares fits to the data with eqn (3.18) and the full lines are fits with eqn (3.23). Inset: symbols are SLS data recorded at $283 \mathrm{~K}$. The full line is the best fit with the Carnahan-Starling expression for hard spheres.

a first attempt we fitted the experimental data with $S(Q=0)$ according to eqn (1.9) with $\gamma$ as the only fitting parameter. To account for the increasing intercept we used the aggregation number of the micelles, $N$, as an additional parameter, which eventually yields

$$
M_{\text {app }}=N M_{\text {mono }} \mathrm{S}(Q=0)
$$

Here $M_{\text {mono }}$ is the molar mass of the surfactant. In Fig. 1 we have plotted experimental $M_{\text {app }} v s . c$ data together with the best fits with eqn (3.18). For the calculation of the fitting curves the experimental concentrations were converted to volume fractions by dividing $c$ by an effective density of $1 \mathrm{~g}$ $\mathrm{mL}^{-1}$. Eqn (3.18) yields very good fits to the experimental data for all temperatures. However, it turns out to be a coincidence since the maximum position of the calculated $M_{\text {app }} v s . c$ curves inevitably approaches a value of $c=0.13 \mathrm{~g} \mathrm{~mL}^{-1}$ with increasing temperature. In this respect the model is not appropriate to fit the data from other surfactant systems. As an example, we show the experimental data from aqueous $\mathrm{C}_{8} \mathrm{E}_{4}$ solutions in Fig. 2 where the maximum position of the experimental $M_{\text {app }} v s . c$ curves occurs significantly below $0.1 \mathrm{~g} \mathrm{~mL}^{-1}$.

This discrepancy can be removed if we allow for a slight anisometry of the micellar shape by converting the experimental surfactant mass concentration, $c$, properly into the micellar volume fraction, $\phi$, which is used as the independent variable in the fitting function. Both quantities are related to the number density of the monomeric surfactant, $\mathscr{N}=N_{\text {surf }} /$ $V$, by

$$
c=\frac{m}{V}=\mathscr{N} \frac{M_{\text {mono }}}{N_{\mathrm{A}}}
$$

and

$$
\phi=\frac{V_{\mathrm{mic}}}{V}=\mathscr{N} \frac{4 \pi a_{\mathrm{eff}}^{3}}{3 N} .
$$

Here we applied a power law

$$
N=N_{0} \phi^{\nu}
$$




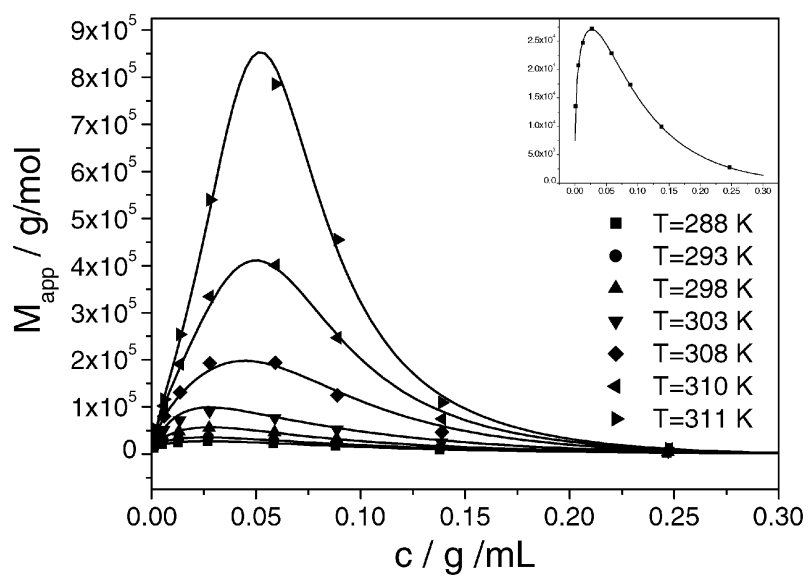

Fig. 2 Static light scattering data from solutions of $\mathrm{C}_{8} \mathrm{E}_{4}$ in water as $M_{\text {app }}$ vs. $c$ (symbols) recorded at temperatures as indicated in the legend. Inset: symbols are SLS data recorded at $288 \mathrm{~K}$. The full lines are fits with eqn (3.23).

to account for micellar growth with concentration. Accordingly, the effective radius has to scale with concentration as $a_{\mathrm{eff}}=a_{0} \phi^{v / 3}$. Consequently, the volume fraction is related to mass concentration by

$$
\phi=c \frac{4 \pi a_{\mathrm{eff}}^{3} N_{\mathrm{A}}}{3 N M_{\text {mono }}} \equiv c \mathscr{K}
$$

where $N_{\mathrm{A}}$ is Avogadro number. Introducing eqn (3.22) into eqns (1.9) and (3.18) yields

$$
M_{\text {app }}=N M_{\text {mono }}\left[\frac{(1+2 c \mathscr{K})^{2}+(c \mathscr{K})^{3}(c \mathscr{K}-4)}{(1-c \mathscr{K})^{4}}-2 \gamma c \mathscr{K}\right]^{-1} .
$$

as the final model function, where the particle radius has to be replaced by $a_{\text {eff }}$ in the expression for $\gamma$ and the aggregation number follows from eqn (3.21). Note that $\mathscr{K}^{-1}$ has the dimension of a density, which takes care of the necessary conversion from experimental concentrations to volume fractions.

The full lines in Fig. 1 and 2 have been obtained by nonlinear least square fitting of eqn (3.23) to the experimental data where $N_{0}, a_{0}$, the Hamaker constant, $A_{\mathrm{H}}, d_{\mathrm{c}}$, and $L_{\mathrm{c}}$ were the floating parameters. From the fitting parameters which are listed in Table 1, we calculated the effective pair interaction according to eqns (1.6) and (1.7). The resulting interaction potentials between two $\mathrm{C}_{6} \mathrm{E}_{4}$ micelles at three selected temperatures are depicted in Fig. 3. The corresponding potentials for the $\mathrm{C}_{8} \mathrm{E}_{4}$ system are shown in Fig. 4.

As already mentioned, the scattering data from the $\mathrm{C}_{6} \mathrm{E}_{4}$ solutions at low temperatures can be described by the Carnahan-Starling equation for hard spheres (eqn (1.9) with $\gamma=0$, see inset of Fig. 1). Accordingly, the interaction potential obtained from the fitting with eqn (3.23) is zero down to contact at low temperature. With increasing temperature attractions set in and the Hamaker constants attain finite values, which increase slightly with temperature. The corresponding Van der Waals attraction given by eqn (1.6) is
Table 1 Parameters from the non-linear least squares fitting of eqn (3.23) to the experimental $M_{\text {app }}$ vs. $c$ data of $\mathrm{C}_{m} \mathrm{E}_{4}$ surfactants in aqueous solution. The brush heights $H_{\mathrm{b}}$ in the last column were calculated for the compositions at the minima of the coexistence curves

\begin{tabular}{lrllllll}
\hline$T /{ }^{\circ} \mathrm{C}$ & $N_{0}$ & $a_{0} / \mathrm{nm}$ & $\nu$ & $A_{\mathrm{H}} / k_{\mathrm{B}} T$ & $L_{\mathrm{c}} / \mathrm{nm}$ & $d_{\mathrm{c}} / \mathrm{nm}^{2}$ & $H_{\mathrm{b}} / \mathrm{nm}$ \\
\hline $\mathrm{C}_{6} \mathrm{E}_{4}$ & & & & & & & \\
10 & 34 & 1.59 & 0 & 0 & 0.74 & 0.31 & 0.31 \\
30 & 58 & 1.83 & 0 & 0 & 0.53 & 0.26 & 0.19 \\
40 & 62 & 1.87 & 0 & 0.59 & 0.43 & 1.4 & 0.1 \\
50 & 100 & 2.18 & 0.015 & 0.63 & 0.17 & 0.28 & 0.06 \\
55 & 219 & 2.89 & 0.15 & 0.65 & 0.12 & 0.15 & 0.03 \\
58 & 393 & 3.53 & 0.18 & 0.68 & 0.044 & 0.01 & $2 \cdot 10^{-3}$ \\
60 & 587 & 4.01 & 0.15 & 0.70 & 0.019 & 0.027 & $7 \cdot 10^{-3}$ \\
& & & & & & & \\
$\mathrm{C}_{8} \mathrm{E}_{4}$ & & & & & & & \\
15 & 232 & 3.51 & 0.94 & 0.24 & 1.12 & 0.08 & 0.19 \\
20 & 341 & 4.00 & 1.14 & 0.31 & 1.07 & 0.09 & 0.20 \\
25 & 498 & 4.62 & 1.09 & 0.33 & 0.89 & 0.11 & 0.19 \\
30 & 2266 & 8.26 & 0.96 & 0.54 & 0.33 & 0.39 & 0.17 \\
35 & 4811 & 10.88 & 0.57 & 0.58 & 0.20 & 0.04 & 0.023 \\
37 & 6687 & 12.22 & 0.65 & 0.60 & 0.01 & 0.03 & $10^{-3}$ \\
38 & 10671 & 14.47 & 0.68 & 0.67 & 0.009 & 0.68 & 0.026 \\
\hline
\end{tabular}

sufficient to cause a minimum in the interaction potential of significant depth. The range of this well is only about a few percent of the particle diameter. For the $\mathrm{C}_{8} \mathrm{E}_{4}$ system we find a pronounced maximum in the $M_{\text {app }} v s$. $c$ curve even at the lowest experimental temperature as is shown in the inset of Fig. 2. In this case, the interaction potentials show an attractive well at all temperatures, which increases in depth and range with increasing temperature.

A qualitatively different scattering behavior is observed for the group of $\mathrm{C}_{m} \mathrm{E}_{8}$ systems. In all cases the $S(Q=0)$ vs. $c$-curves decrease monotonously with increasing concentration. In the case of the $\mathrm{C}_{10} \mathrm{E}_{8}$ solutions, the scattering data could be force fitted with eqn (3.23) (dotted lines in Fig. 5) which was impossible for the $\mathrm{C}_{12} \mathrm{E}_{8^{-}}$and $\mathrm{C}_{16} \mathrm{E}_{8}$ data. Since these systems show an increasing tendency to form cylindrical micelles, we fitted these data with eqn (1.16). We applied a routine, which fitted the data sets collected at different temperature simultaneously, using $\beta$ as a global parameter, while

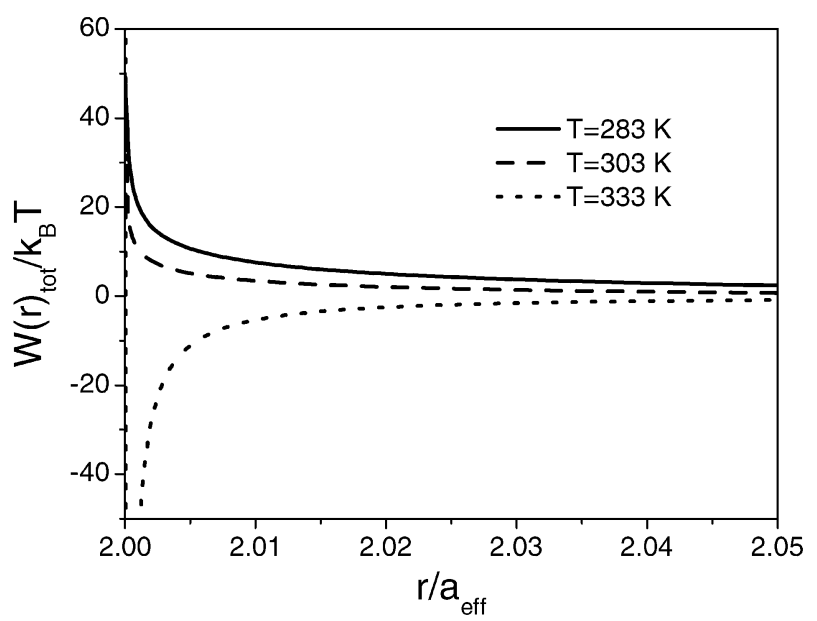

Fig. 3 Pair interaction potentials in aqueous $\mathrm{C}_{6} \mathrm{E}_{4}$ solution at various temperatures as indicated in the legend. The curves were calculated introducing the fit parameters from Table 1 into eqns (1.6) and (1.7). 


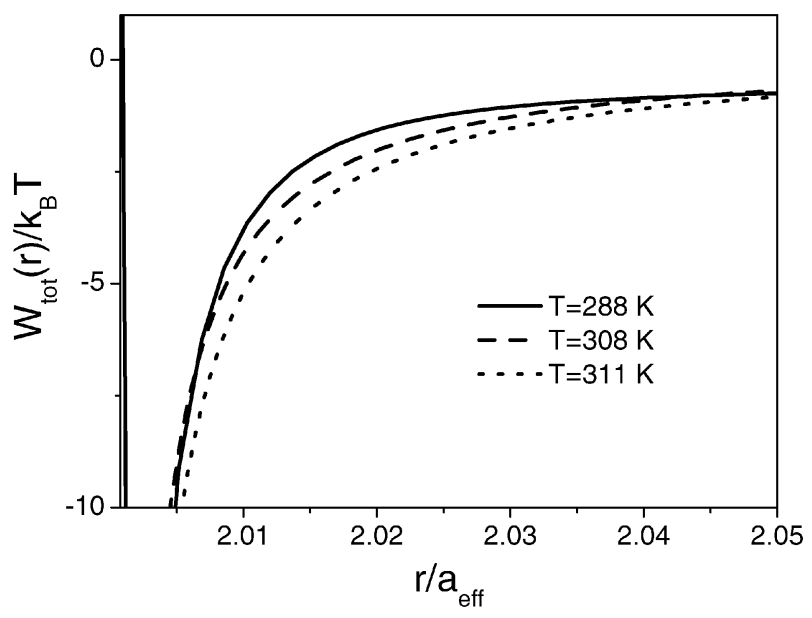

Fig. 4 Pair interaction potentials in micellar $\mathrm{C}_{8} \mathrm{E}_{4}$ solution at various temperatures as indicated in the legend. The curves were calculated introducing the fit parameters from Table 1 into eqns (1.6) and (1.7).

$N$ and $\chi_{\text {wm }}$ were optimized locally. The experimental data and the resulting best fitting curves are shown in Figs. 5, 7 and 8 and the fitting parameters are listed in Table 2.

\subsection{Fitting parameters and coexistence curves}

The fitting parameters listed in Table 1 can be divided in two groups, the one of which $\left(a_{0}, N_{0}\right.$ and $\left.\nu\right)$ describe the dependence of the micellar size on temperature and concentration. The other set $\left(A_{\mathrm{H}}, L_{\mathrm{c}}\right.$ and $\left.d_{\mathrm{c}}\right)$ reflects the variation of the interaction potential with temperature.

For both $\mathrm{C}_{m} \mathrm{E}_{4}$ surfactants micellar growth occurs with increasing temperature, though much more pronounced for the $\mathrm{C}_{8} \mathrm{E}_{4}$ system. This is reflected in the temperature dependence of the parameters $a_{0}$ and $N_{0}$. Further the parameter $\nu$, which is a qualitative measure for the tendency of the micelles to grow with surfactant concentration is much larger for $\mathrm{C}_{8} \mathrm{E}_{4}$. This is in accordance with Israelachvili's concept of packing parameters ${ }^{27}$ according to which the tendency to form cylind-

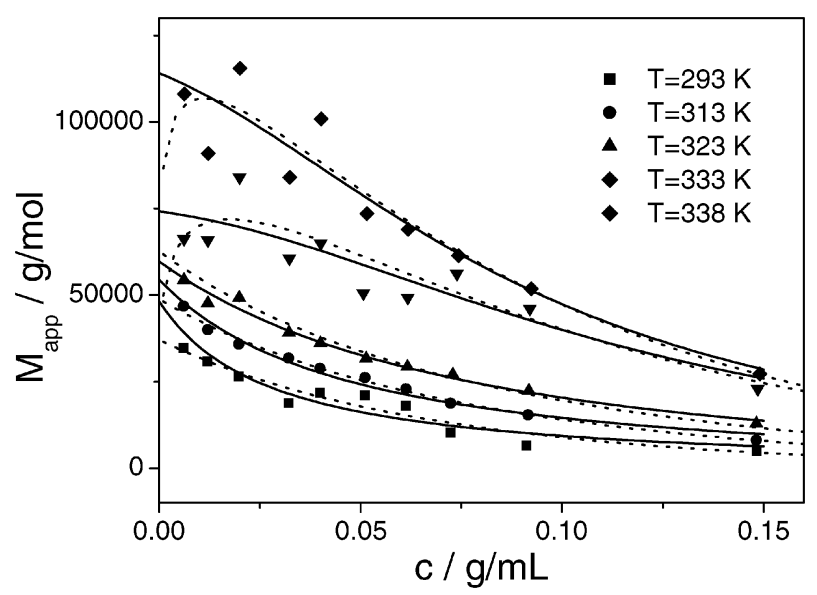

Fig. 5 Static light scattering data from solutions of $\mathrm{C}_{10} \mathrm{E}_{8}$ in water as $M_{\text {app }}$ vs. $c$ (symbols) recorded at temperatures as indicated in the legend. The dotted lines are fits with eqn (3.23) and the full lines represent best fits with eqn (1.16).
Table 2 Parameters from the non-linear least squares fitting of eqn (1.16) to the experimental $M_{\text {app }}$ vs. $c$ data of $\mathrm{C}_{m} \mathrm{E}_{8}$ surfactants in aqueous solution

\begin{tabular}{lccccc}
\hline $\mathrm{C}_{10} \mathrm{E}_{8}$ & & & & & \\
\hline$T /{ }^{\circ} \mathrm{C}$ & 20 & 40 & 50 & 60 & 65 \\
\hline$\beta$ & 1.80 & & & & \\
$N$ & 38 & 43 & 47 & 59 & 90 \\
$\chi_{\text {wm }}$ & 0.01 & 0.23 & 0.35 & 0.48 & 0.48 \\
\hline
\end{tabular}

\begin{tabular}{lccccc}
\hline $\mathrm{C}_{12} \mathrm{E}_{8}$ & & & & & \\
\hline$T /{ }^{\circ} \mathrm{C}$ & 20 & 40 & 50 & 60 & 65 \\
\hline$\beta$ & 2.48 & & & & \\
$N$ & 88 & 90 & 113 & 332 & 493 \\
$\chi_{\text {wm }}$ & 0.32 & 0.41 & 0.49 & 0.50 & 0.51 \\
\hline
\end{tabular}

\begin{tabular}{lcccccc}
\hline $\mathrm{C}_{16} \mathrm{E}_{8}$ & & & & & & \\
\hline$T /{ }^{\circ} \mathrm{C}$ & 20 & 30 & 40 & 45 & 50 & 53 \\
\hline$\beta$ & 9.21 & & & & & \\
$N$ & 26 & 38 & 328 & 789 & 2326 & 3845 \\
$\chi_{\mathrm{wm}}$ & 0.21 & 0.32 & 0.37 & 0.39 & 0.41 & 0.42 \\
\hline
\end{tabular}

rical micelles increases if the volume of the hydrophopbic tail increases at constant size of the hydrophilic head group.

The fitted values for the Hamaker constant $A_{\mathrm{H}}$ and the contour length $L_{\mathrm{c}}$ of the brush chains also have a very clear temperature trend. On the other hand, there is no such trend to be observed for the cross-sectional diameter, $d_{\mathrm{c}}$, of the chains. However, the quality of the fits is the least sensitive to this parameter. Some test calculations show that changing $d_{\mathrm{c}}$ by even an order of magnitude does not change the results significantly if the Hamaker constant $A_{\mathrm{H}} \gtrsim k_{\mathrm{B}} T / 3$. The latter increases continuously with temperature, leading to an increasing attractive interaction, while the brush repulsion is weakened with increasing temperature. This is reflected in the temperature dependence of $L_{\mathrm{c}}$ and the resulting brush height $H_{\mathrm{b}}$, which both decrease by orders of magnitude when the system approaches the coexistence curve. As shown in section 2.1 the location of these curves can be calculated from eqn (1.10). For this purpose we calculated $\gamma(c)$ at the experimental temperatures and extrapolated these data to higher temperatures at constant $c$. In Fig. 6 the coexistence curves calculated with the extrapolated $\gamma$ values are shown together with the experimental data by Schubert et al. ${ }^{5}$ It is evident that the calculated curves match the experimental data very well, even in the vicinity of the critical point.

This very encouraging result motivated us to test the model with other surfactants. As a first choice we took $\mathrm{C}_{12} \mathrm{E}_{8}$ and $\mathrm{C}_{16} \mathrm{E}_{8}$, as these surfactans should, according to their chemical composition, have the same size ratio between their hydrophobic and hydrophilic moieties as the investigated $\mathrm{C}_{m} \mathrm{E}_{4}$ surfactants. Accordingly, one would expect to find a rather similar micellar shape and probably also similar interaction potentials. However, as can be seen from Figs. 7 and 8 the scattering behavior is completely different from that of the shorter homologous surfactants. Very recent literature data on $\mathrm{C}_{16} \mathrm{E}_{8}$ suggest that this surfactant forms cylindrical micelles, ${ }^{17}$ and we therefore chose to fit the scattering data by eqn (1.16), 


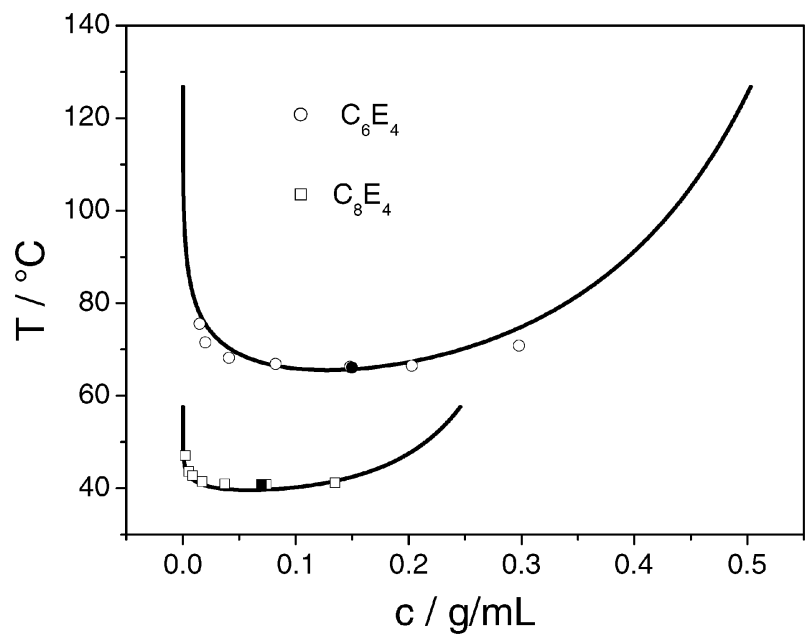

Fig. 6 Liquid/liquid coexistence curves of aqueous $\mathrm{C}_{m} \mathrm{E}_{4}$ solution. Symbols are experimental data $^{5}$ and the full line are calculated introducing the fit parameter from Table 1 into eqn (1.10).

which is based on the analogy between cylindrical micelles and polymers. Further we tried both approaches for the $\mathrm{C}_{10} \mathrm{E}_{8}$ system, which we expected to form aggregates in the intermediate range between long cylindrical and spherical micelles. As shown in Fig. 5 the experimental scattering data from $\mathrm{C}_{10} \mathrm{E}_{8}$ solutions can be force fitted with eqn (3.23). However, the resulting interaction parameters would lead to a coexistence curve, the critical point of which lies far above $100{ }^{\circ} \mathrm{C}$. This implies that the model does not match the physical reality, and that the parameters from the cylidrical micelles model shall be discussed for all three $\mathrm{C}_{m} \mathrm{E}_{8}$ systems.

It can be seen from Table 2 that the parameter $\beta$, which is a measure for the size ratio of a surfactant molecule over a water molecule increases with the chain length of the surfactant. In all three cases we observe a similar trend for the dependence of $N$ and $\chi$ on temperature. The parameter $N$ increases with temperature, indicating an increase of the aggregation number that is the length of the cylindrical micelles. This growth is more pronounced the longer the surfactant molecule is, which

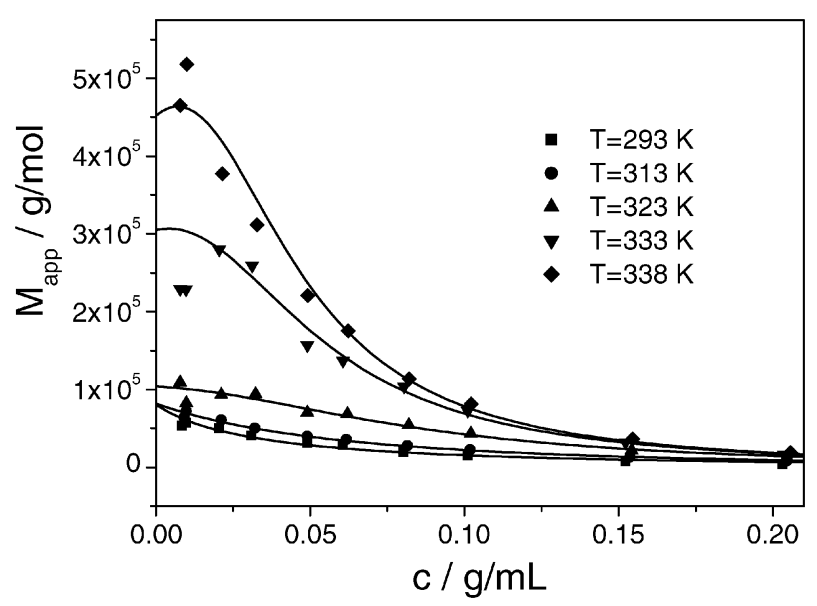

Fig. 7 Static light scattering data from solutions of $\mathrm{C}_{12} \mathrm{E}_{8}$ in water as $M_{\text {app }}$ vs. $c$ (symbols) recorded at temperatures as indicated in the legend. The full lines are fits with eqn (1.16).

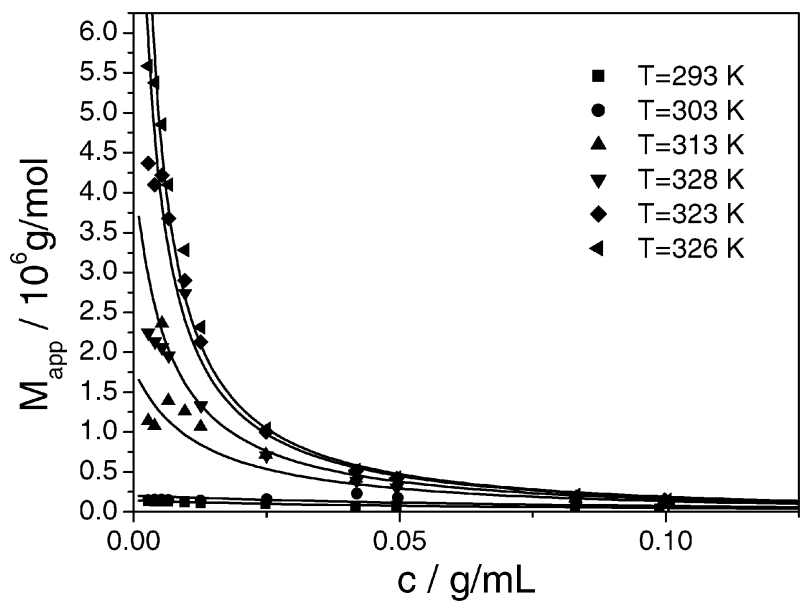

Fig. 8 Static light scattering data from solutions of $\mathrm{C}_{16} \mathrm{E}_{8}$ in water as $M_{\text {app }}$ vs. $c$ (symbols) recorded at temperatures as indicated in the legend. The full lines are fits with eqn (1.16).

again illustrates that the tendency to form cylindrical structures growths with increasing length of the hydrophobic tail. In all three cases the $\chi_{\text {wm }}$ parameter approaches a value of $\chi_{\mathrm{wm}} \approx 0.5$ above which liquid/liquid phase separation occurs at large $N$.

The location of the liquid/liquid coexistence curves of the $\mathrm{C}_{m} \mathrm{E}_{8}$ solutions was calculated using eqn (1.17). The results are plotted in Fig. 9 together with literature data for the corresponding critical points. The coexistence curves become increasingly narrow and their critical points shift towards lower surfactant concentration and lower temperature with increasing $m$. This general trend is in accordance with the coexistence curves as approximated by Inoue et al. ${ }^{50}$ These authors neglected the chemical potential of the surfactant in the dilute phase which makes the calculation of the coexistence curves inaccurate at least near the minimum of the curve. We find that our calculated critical points agree increasingly well with the experimental data ${ }^{46}$ on passing on from $\mathrm{C}_{10} \mathrm{E}_{8}$ to $\mathrm{C}_{16} \mathrm{E}_{8}$. This shows that the theoretical model describes the behavior of

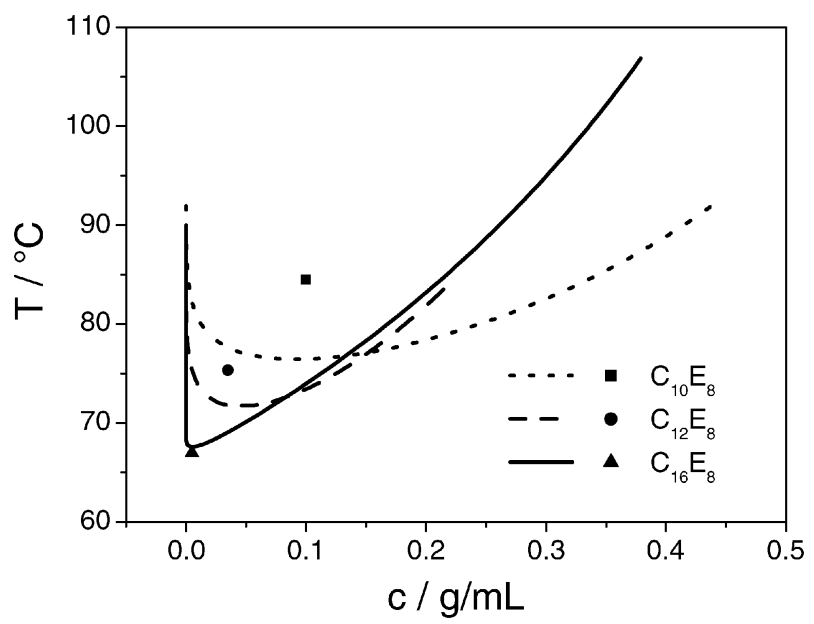

Fig. 9 Liquid/liquid coexistence curves of aqueous $\mathrm{C}_{m} \mathrm{E}_{8}$ solution. Symbols are experimental $\mathrm{data}^{46}$ and the full line are calculated introducing the fit parameter from Table 2 into eqn (1.17). 
cylindrical $\mathrm{C}_{16} \mathrm{E}_{8}$ micelle reasonably well, while the agreement becomes worse for the shorter chain surfactants. This is to be expected since the theory is designed for very long cylindrical micelles.

\section{Conclusions}

We presented scattering data from two sets of micelles, i.e. $\mathrm{C}_{m} \mathrm{E}_{4}$ and $\mathrm{C}_{m} \mathrm{E}_{8}$, which exhibit completely different scattering behavior. The $\mathrm{C}_{m} \mathrm{E}_{4}$ systems can be very well described by a model of spherical or nearly spherical micelles interacting via an attractive Van der Waals potential and a stabilizing repulsive brush potential in addition to a hard core excluded volume interaction. On the other hand the behavior of the members of the $\mathrm{C}_{m} \mathrm{E}_{8}$ family can not be described by this model, even though the investigated $\mathrm{C}_{m} \mathrm{E}_{8}$ surfactants had exactly twice the chain length as the $\mathrm{C}_{m} \mathrm{E}_{4}$ surfactants. The $\mathrm{C}_{m} \mathrm{E}_{8}$ systems could be described with the model of cylindrical micelles interacting via an effective excluded volume between the surfactant molecules following a modified Flory-Huggins theory. This leads us to conclude that not only the relative sizes of hydrophobic to hydrophilic moiety have an influence on the micellar shape, but also the absolute chain length plays an important role.

We have demonstrated that we can accurately predict the liquid/liquid coexistence curves of $\mathrm{C}_{m} \mathrm{E}_{n}$ solutions from scattering data for systems which contain micelles that are sufficiently close to spherical shape. In the transition regime between spherical micelles the agreement between experimental data and coexistence curves deduced from scattering data is less accurate. However, in the second limiting case of very long cylindrical micelles we find again excellent accordance.

\section{Acknowledgements}

We thank R. Strey for supplying the experimental data for the location of the liquid/liquid coexistence curves of the $\mathrm{C}_{m} \mathrm{E}_{4}$ solutions and $\mathrm{P}$. Holmqvist for many helpful discussions.

\section{References}

1 For an overview see Surfactant Science Series Vols. 1, 19, 23, 60 and 72, Marcel Dekker, New York and Basel.

2 R. R. Balmbra, J. S. Clunie, J. M. Corkill and J. F. Goodman, Trans. Faraday Soc., 1962, 58, 1661.

3 D. J. Mitchell, G. J. T. Tiddy, L. Waring, T. Bostock and M. P. McDonald, J. Chem. Soc., Faraday Trans. 1, 1983, 79, 975.

4 J. Sjöblom, P. Stenius and J. Danielsson, in Phase Equilibria of Nonionic Surfactants and the Formation of Microemulsions, ed. M. Schick, Nonionic Surfactants Physical Chemistry; Surfactant Science Series 23, Marcel Dekker, New York and Basel, 1987.

5 K. V. Schubert, R. Strey and M. Kahlweit, J. Colloid Interface Sci., 1991, 141, 21.

6 J. B. Hayter and M. Zulauf, Colloid Polym. Sci., 1982, 260, 1023.

7 W. Brown, R. M. Johnsen and P. Stilbs, J. Phys. Chem., 1983, 87, 4548.

8 M. Zulauf, K. Weckstrøm, J. B. Hayter, V. Degiorgio and M. Corti, J. Phys. Chem., 1985, 89, 3411.

9 W. Brown and R. Rymdn, J. Phys. Chem., 1987, 91, 3565.
10 W. H. Richtering, W. Burchard, E. Jahns and H. Finkelmann, J. Phys. Chem., 1988, 92, 6032.

11 W. Brown, Z. Pu and R. Rymdn, J. Phys. Chem., 1988, 92, 6086.

12 H. Strunk, P. Lang and G. H. Findenegg, J. Phys. Chem., 1994, 98, 11557.

13 P. Schurtenberger, C. Cavaco, F. Tiberg and O. Regev, Langmuir, 1996, 12, 2894.

14 J. Brunner-Popela and O. Glatter, J. Appl. Crystallogr., 1997, 30, 431.

15 U. Menge, P. Lang and G. H. Findenegg, J. Phys. Chem. B, 1999, 103, 5768.

16 O. Glatter, G. Fritz, H. Lindner, J. Brunner-Popela, R. Mittelbach, R. Strey and S. U. Egelhaaf, Langmuir, 2000, 16, 8692.

17 N. Hamada and Y. Einaga, J. Phys. Chem. B, 2005, 109, 6990.

18 V. J. Degiorgio and M. Corti, Phys. Rev. Lett., 1980, 45, 1045.

19 V. J. Degiorgio and M. Corti, J. Phys. Chem., 1981, 85, 1442.

20 A. Zielesny, L. Belkoura and D. Woermann, Ber. Bunsen-Ges. Phys. Chem., 1994, 98, 195.

21 M. Lesemann, L. Belkoura and D. Woermann, Ber. Bunsen-Ges. Phys. Chem., 1994, 98, 579.

22 M. Zulauf and J. P. Rosenbusch, J. Chem. Phys., 1983, 87, 856.

23 B. Lindman and H. Wennerstrøm, J. Phys. Chem., 1991, 95, 6053.

24 C. Stubenrauch, M. Nydén, G. H. Findenegg and B. Lindman, J. Phys. Chem., 1996, 100, 17028.

25 D. J. Cebula and R. H. Ottewill, Colloid Polym. Sci., 1982, 216, 1118.

26 (a) R. Strey and A. Pakusch, in Surfactants in Solution, ed. K. L. Mittal and P. Bothorel, Plenum Press, New York, 1986, vol. 4, p. 465 .

27 J. N. Israelachvili, D. J. Mitchell and B. W. Ninham, J. Chem. Soc., Faraday Trans. 1, 1976, 72, 1525.

28 P. Schurtenberger and C. Cavaco, J. Phys. II, 1993, 3, 1279.

29 T. Ohta and Y. Oono, Phys. Lett., 1982, 89A, 460.

30 R. J. Baxter, J. Chem. Phys., 1968, 49, 2270.

31 C. Robertus, J. G. H. Joosten and Y. K. Levine, Phys. Rev. A, 1990, 42, 4820.

32 S. H. Chen, J. Rouch, F. Sciortino and P. Tartaglia, J. Phys.: Condens. Matter, 1994, 6, 10855.

33 P. Lang and O. Glatter, Langmuir, 1996, 12, 1193.

34 J. W. Jansen, C. G de Kruif and A. Vrij, J. Colloid Interface Sci., 1986, 114, 471.

35 J. K. Percus and G. J. Yevick, Phys. Rev., 1958, 110, 1.

36 J. F. Carnahan and K. E. Starling, J. Chem. Phys., 1969, 51, 635.

37 S. M. Walas, Phase Equilibria in Chemical Engineering, Butterworth, Boston, 1989.

38 A. Vrij, E. A. Nieuwenhuis, H. M. Fijnaut and W. G. M. Agterof, Faraday Discuss., 1978, 65, 101.

39 Textbooks of colloidal science e.g. J. Lyklema, Fundamentals of Interface and Colloidal Science, Academic Press, London, 1991, vol. 1, ch. 4.

40 S. Alexander, J. Phys. (France), 1977, 38, 983.

41 P. G. DeGennes, Adv. Colloid Interface Sci., 1987, 27, 189.

42 C. N. Likos, K. A. Vaynberg, H. Löwen and N. J. Wagner, Langmuir, 2000, 16, 4100.

43 R. Kjellander and E. Florin, J. Chem. Soc., Faraday Trans. 1, 1981, 77, 2053.

44 A. Vrij and R. Tuinier, Structure of concentrated colloidal dispersions, in Fundamentals of Interface and Colloid Science, volume IV: Particulate Colloids, ed. J. Lyklema, Elsevier, Amsterdam, 2005, ch. 5 .

45 L. A. M. Rupert, J. Colloid Interface Sci., 1992, 153, 92.

46 N. M. van Os, J. R. Haak and L. A. M. Rupert, Physico-Chemical Properties of Selected Anionic, Cationic and Nonionic Surfactants, Elsevier, Amsterdam, London, New York, Tokyo, 1993.

47 J. C. Hansen, D. Maier, J. Honerkamp, W. Richtering, F. M. Horn and H. Senff, J. Colloid Interface Sci., 1999, 215, 72.

48 M. B. Huglin, Light scattering from polymer solutions, Academic Press, London, 1972.

49 T. Matsumoto and H. Zenkoh, Colloid Polym. Sci., 1990, 268, 536.

50 T. Inoue, H. Ohmura and D. Murata, J. Colloid Interface Sci., 2003, 258, 374. 